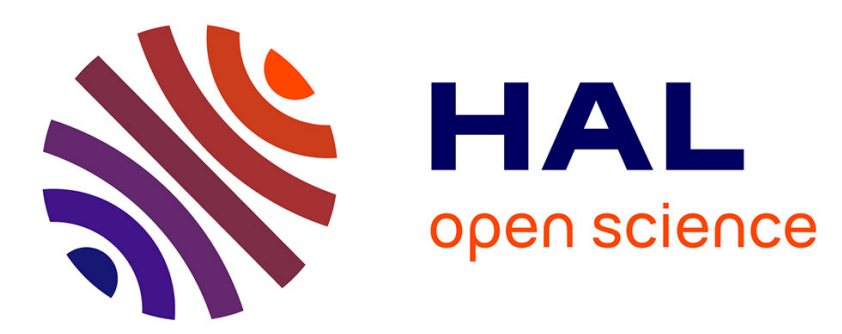

\title{
Formalisation des processus de la veille épidémiologique des maladies infectieuses
}

Gaoussou Camara, Sylvie Després, Moussa Lo

\section{To cite this version:}

Gaoussou Camara, Sylvie Després, Moussa Lo. Formalisation des processus de la veille épidémiologique des maladies infectieuses. Reconnaissance de Formes et Intelligence Artificielle (RFIA) 2014, Jun 2014, France. hal-00989220

\section{HAL Id: hal-00989220 https://hal.science/hal-00989220}

Submitted on 9 May 2014

HAL is a multi-disciplinary open access archive for the deposit and dissemination of scientific research documents, whether they are published or not. The documents may come from teaching and research institutions in France or abroad, or from public or private research centers.
L'archive ouverte pluridisciplinaire HAL, est destinée au dépôt et à la diffusion de documents scientifiques de niveau recherche, publiés ou non, émanant des établissements d'enseignement et de recherche français ou étrangers, des laboratoires publics ou privés. 


\title{
Formalisation des processus de la veille épidémiologique des maladies infectieuses
}

\author{
G. Camara ${ }^{1,2,3} \quad$ S. Despres ${ }^{1} \quad$ M. Lô $\hat{o}^{2}$ \\ ${ }^{1}$ INSERM, U1142, LIMICS, F-75006, Paris, France \\ ${ }^{2}$ LANI, Université Gaston Berger, B.P. 234, Saint-Louis du Sénégal \\ ${ }^{3}$ Université Alioune Diop de Bambey, B.P. 30, Bambey, Sénégal \\ gaoussou.camara@uadb.edu.sn \\ sylvie.despres@univ-paris13.fr \\ moussa.lo@ugb.edu.sn
}

\begin{abstract}
Résumé
La construction d'un système de veille épidémiologique vise à mettre en place un dispositif de prédiction de l'impact d'événements constituant des facteurs de risque de propagation d'une maladie et d'élaboration des plans d'action pour en contrôler son évolution. Ainsi, la modélisation d'un tel système consiste principalement à modéliser deux processus encapsulés : le processus de veille qui a pour objet d'étude le processus de propagation du phénomène épidémiologique. Dans ce papier, nous nous focalisons sur la formalisation d'une ontologie du processus de veille. La construction de cette ontologie permet de représenter l'ordonnancement des différentes phases de l'activité de veille, les conditions de déclenchement de chaque phase, les ressources mises en cuvre dans chaque phase, etc. Elle permet ainsi d'automatiser, afin de planifier, superviser et contrôler, la mise en cuvre des processus de la veille.
\end{abstract}

\section{Mots Clef}

Veille, épidémiologie, ontologie de processus, modélisation, formalisation

\begin{abstract}
Epidemiological monitoring is implemented to foresee the consequences of events considered as risk factor of disease spreading and to suggest action plans for controlling its evolution. Thus, epidemiological monitoring system modeling mainly focuses on representing two encapsulated processes: the monitoring process having the epidemiological phenomenon spreading as subject of study. In this paper, we focus on formalizing the monitoring process ontology. The building of this ontology allows us representing the execution of the monitoring phases, the launching condition of each phase, the resources involved in each of these phases, etc. Thus, it permits us to automate (in order to plan), supervise and control the execution of the monitoring processes.
\end{abstract}

\section{Keywords}

Monitoring, epidemiology, process ontology, modeling, formalization

\section{Introduction}

La veille épidémiologique est mise en œuvre à la fois, pour prédire l'impact d'événements constituant des facteurs de risque de propagation d'une maladie et pour suggérer des plans d'action pour en contrôler son évolution. Elle consiste à surveiller, détecter des événements, collecter des données, analyser ces données (par la simulation par exemple), interpréter les résultats de l'analyse, prendre des décisions et évaluer les décisions prises [1]-[3]. Ainsi, la modélisation d'un système de veille épidémiologique consiste principalement à modéliser deux processus encapsulés : le processus de veille qui a pour objet d'étude le processus de propagation du phénomène épidémiologique. Le papier est axé sur la formalisation du processus de la veille et non sur le processus de propagation du phénomène épidémiologique.

La construction d'une ontologie des processus de veille permet de représenter l'ordonnancement des différentes phases de l'activité de veille, les conditions de déclenchement de chaque phase, les ressources mises en œuvre dans chaque phase et leurs résultats (sortie ou effet sur les autres phases ou entités du domaine). Contrairement à l'ontologie de domaine de la veille, l'ontologie des processus met l'accent sur le mode de déroulement des activités au cours du temps. Par conséquent, elle peut être utilisée pour planifier les processus tels que les décisions, en contrôler leur évolution et réaliser des simulations.

Dans la suite de l'article, nous introduisons la modélisation ontologique des processus en section 2. Les sections 3 et 4 présentent respectivement les processus de veille et ceux de la veille épidémiologique des maladies infectieuses. La modélisation et la formalisation du processus de veille épidémiologique sont présentées dans les sections 5 et 6 . L'ontologie de la veille épidémiologique est conçue sous forme d'un patron de structuration en section 7 et un exemple de son extension pour la veille de la schistosomiase au Sénégal est donné en section 8 . Nous concluons en section 9 . 


\section{Modélisation ontologique des processus}

Les ontologies de processus fournissent une représentation des connaissances sur les processus afin d'en faciliter leur partage. Les processus se distinguent des connaissances de domaine par la prise en compte de la persistance des entités dans le temps [4], [5]. Les ontologies de domaine, notamment les ontologies de fondement, fournissent une première catégorisation des entités du monde réel en fonction de leur mode de persistance :

- les endurants/continuants: entités sans partie temporelle comme les objets

- les perdurants/occurrents : entités évoluant dans le temps avec différentes phases comme les processus

Les ontologies de processus donnent une spécification du mode de déroulement des perdurants/occurrents dans le temps. Cette description porte sur l'enchaînement des phases constituant le processus, les entrées et les sorties, les endurants/continuants participant à sa dynamique, les effets des occurrences de ce processus sur les autres entités du domaine.

Bien que les définitions des ontologies de processus soient proches de celles des ontologies de tâches, ces dernières peuvent être vues comme des ontologies de processus se restreignant au processus de résolution de problème [6], [7]. Les ontologies de processus peuvent par conséquent être envisagées comme une généralisation des ontologies de tâches. Cette distinction nous conduit d'ailleurs à distinguer les processus de gestion des processus naturels. Les processus de gestion sont ceux établis par les humains, et sont par conséquent contrôlables. Par exemple, le processus de fabrication d'un produit industriel, le processus de traitement d'une maladie, le processus de gestion d'une catastrophe naturelle, le processus de résolution d'une équation du second degré, etc. Cette catégorie inclut également les procédures qui consistent à associer les phases de façon à aboutir à un résultat escompté. Les processus naturels sont ceux dont les conditions de déroulement ne sont pas établies par les humains mais qui existent naturellement, par exemple le processus de changement climatique, le processus de propagation d'une maladie, le processus du cycle de l'eau, le processus de croissance d'une plante, etc. Même s'ils ne sont pas entièrement contrôlables, les humains peuvent agir sur leur déroulement pour en changer leur comportement futur. Ces actions se traduisent par exemple par le contrôle et la prévention de la propagation d'une maladie, l'accélération de la croissance d'une plante, la diminution de l'émission des gaz à effet de serre pour ralentir le réchauffement climatique, etc.

La modélisation ontologique des processus de la veille épidémiologique est guidée par deux objectifs :
- Fournir une description explicite des processus avec un langage de spécification de processus pour faciliter leur interopérabilité et leur partage entre les applications du système de veille.

- Fournir une spécification formelle des processus permettant d'automatiser, afin de planifier, superviser et contrôler, leur mise en œuvre dans le contexte de la veille. La formalisation des processus permet aussi d'expliquer leur occurrence ou leur comportement.

\section{Les processus de veille}

L'étymologie du terme veille est très large. Si la veille est d'abord définie comme l'état de rester éveillé ou de monter une garde, elle est considérée dans le milieu des entreprises comme une activité consistant à étudier des informations stratégiques afin d'anticiper des évènements. Bien que souvent confondu avec la surveillance, la veille va au-delà de celle-ci. Cette confusion conduit à un point de vue réducteur sur la portée de la veille. En effet, la veille ne se limite pas simplement à surveiller les éléments dont une variation peut potentiellement avoir un impact sur un autre phénomène dont on veut maintenir la stabilité ou améliorer son état. La veille est tout un dispositif de surveillance conçu pour agir par anticipation [8]. Dès lors, pour la conception d'un dispositif de veille, il est indispensable de pouvoir répondre aux questions suivantes: Surveiller quoi ? Agir sur quoi ? Dans quel but? Quels que soient les domaines technologique, sismique, sanitaire, etc. où elle s'applique la veille peut toujours être décomposée en quatre principales sous activités : surveillance, analyse, décision et évaluation (cf. Figure 1) [1].

Chacune de ces activités se décompose en sous-activités. Dans cette partie nous nous limiterons à deux niveaux de décomposition pour mettre en évidence les principales sous-activités de la veille. La mise en œuvre de ces activités peut varier dans les différents domaines d'application.

- La surveillance consiste à observer de façon permanente les facteurs dont une variation peut avoir un impact sur la stabilité du phénomène surveillé. La surveillance est ainsi décomposée en deux sous-activités :

- La détection qui consiste à capter toutes les variations sur les facteurs de risque de perturbation du phénomène. Par exemple, un changement de température important ou l'arrivée d'un nouveau concurrent sur le marché doivent être détectés.

- La collecte porte sur les données relatives aux variations des facteurs de risque qui sont nécessaires à l'analyse des conséquences sur le comportement du phénomène. Par 
exemple, les différentes températures mesurées suivant une fréquence temporelle ou les informations sur le concurrent et les caractéristiques des produits mis sur le marché.

- L'analyse consiste à étudier les comportements futurs probables du phénomène en fonction des données collectées suite à un événement détecté. L'analyse est composée des sous-activités suivantes :

- le traitement des données collectées pour prédire les comportements possibles du phénomène suite à la détection de variations des facteurs de risque susceptibles d'influer sur la stabilité du phénomène ;

- l'interprétation consiste à déchiffrer les résultats du traitement pour en tirer un diagnostic sur les risques de perturbation du phénomène ;

- l'alerte prévient, si un risque est identifié, les acteurs concernés sur les évolutions probables du phénomène dans le futur.

- La décision mise en œuvre vise un impact sur le comportement du phénomène en agissant sur les facteurs de risque contrôlables. Les décisions peuvent porter sur les facteurs de risque contrôlables ou agir sur les entités impliquées dans la dynamique du phénomène pour contrer son évolution. La décision est composée des sous-activités suivantes :

- la définition des plans d'action;

- l'évaluation des plans d'action ;

- la sélection de plans d'action ;

- la mise en œuvre de plan d'action.

- L'évaluationdes décisions prises est indispensable pour mesurer leurs impacts et leurs efficacités. L'évaluation est composée des sousactivités suivantes :

- la définition des stratégies d'évaluation ;

o la mise en œuvre de l'évaluation ;

o l'analyse des résultats de l'évaluation.

\section{La veille épidémiologique des maladies infectieuses}

Plusieurs points de vue sur les maladies sont adoptés en médecine selon les études réalisées: (i) l'interaction biologique de l'agent pathogène avec l'organisme (Biologie), (ii) les signes et symptômes chez le patient et son mode de traitement (Clinique), et (iii) le mode de propagation au sein d'une population d'individus et ses facteurs de risque (Epidémiologie). La veille épidémiologique porte essentiellement sur la propagation au niveau des populations d'individus et est enrichie par les points de vue clinique et biologique.

La veille épidémiologique est une activité consistant à surveiller de façon continue un phénomène épidémiologique afin d'anticiper et contrôler son évolution dans le temps dans une population située dans une région géographique donnée. Les connaissances mises en œuvre dans le domaine de la veille épidémiologique sont produites en épidémiologie. Le rôle de l'épidémiologie est d'étudier les différents facteurs intervenant dans l'apparition et la propagation d'une maladie et les moyens à mettre en œuvre pour sa prévention et son contrôle. "L'épidémiologie est une discipline scientifique qui étudie notamment les différents facteurs intervenant dans l'apparition des maladies ou de phénomènes de santé ainsi que leur fréquence, leur mode de distribution, leur évolution et la mise en auvre des moyens nécessaires à la prévention ». [9]. L'épidémiologie permet par conséquent d'enrichir le domaine de connaissances liées à la dynamique de propagation d'une maladie et à ses facteurs de risque. La connaissance de ces caractéristiques d'une maladie permet ainsi la définition des stratégies de veille épidémiologique: les conditions de lancement du processus de détection, les aspects visés par les processus de décision définis, etc.

La veille épidémiologique est modélisée comme une spécialisation du processus de veille dans lequel le processus de traitement figurant dans la phase d'analyse se fait par simulation. La méthode de traitement utilisée dans les systèmes de veille dépend de la nature du phénomène surveillé. La propagation d'une maladie infectieuse étant considérée comme un système complexe [10], la compréhension et l'analyse de son évolution et de sa dynamique nécessitent de recourir à des simulations reproduisant son comportement [11].

Le sous-domaine de la veille comprend les acteurs individuels et organisationnels ainsi que leurs rôles, les ressources exploitées et les différentes phases de sa mise en œuvre. Les acteurs de la veille sont les experts de la maladie ou des domaines liés aux facteurs de risque comme les météorologues. Les acteurs intervenant dans les processus de prise de décision, tels que les pouvoirs publics ou encore les acteurs mettant en œuvre les décisions prises ou les agents de santé publique sont également à considérer. Les ressources de la veille sont constituées des modèles de simulation permettant d'étudier le phénomène épidémiologique, les sources de données utilisées pour les simulations, les logiciels de simulation et autres applications utilisées. Les modèles de simulation sont la reproduction d'une partie de la dynamique réelle du phénomène épidémiologique avec des objectifs précis, de prédiction par exemple. Les logiciels de simulation mettent en œuvre ces modèles pour pouvoir observer l'évolution d'une partie de la réalité du 
système dans le temps et dans l'espace. A ce niveau, les modèles de simulation représentent les entités statiques du sous domaine, tandis que les simulations représentent une dynamique, une sorte de processus se réalisant dans un intervalle de temps bien défini et reproduisant un comportement du phénomène.

\section{Modélisation du processus de veille épidémiologique}

Dans cette première version de l'ontologie, nous modélisons les relations de «precedence » des activités de la veille et les sous-activités des activités complexes. Dans ce cas, la notion d'activité fait référence à la notion de processus. La Figure 1 présente un aperçu des différentes phases de la veille épidémiologique (surveillance, analyse, décision et évaluation) et les sous-activités composant chacune de ces phases. Notez par exemple que le traitement dans la phase d'analyse est réalisé par des simulations du fait de la nature complexe des phénomènes épidémiologiques.

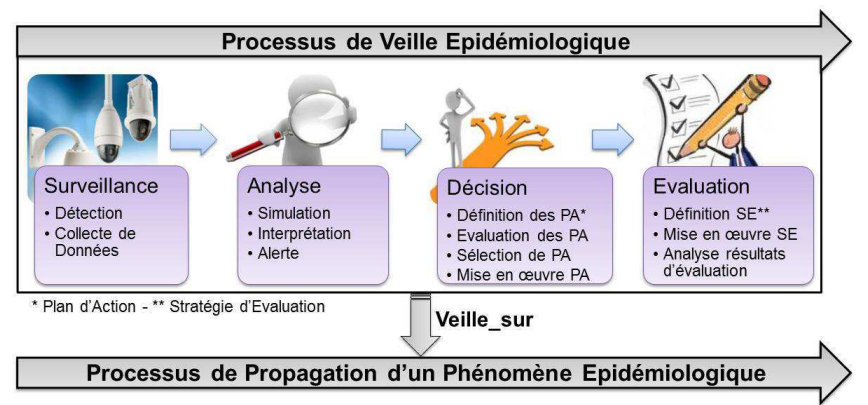

Figure 1 : Les processus de la veille épidémiologique

Les relations de précédence et de composition (partie-de) des activités de la veille sont décrites dans le Tableau 1. La veille est la macro-activité du processus de veille épidémiologique par conséquent elle ne suit et ne fait partie d'aucune activité. La surveillance n'est précédée par aucune activité car elle est la première sous-activité de la veille. Par conséquent, la détection (première sousactivité de la surveillance) n'est précédée d'aucune autre activité. Cependant, la simulation (première sous-activité de l'analyse) est précédée par la collecte (dernière sousactivité de la surveillance).

A ce stade de la modélisation, nous choisissons la relation de précédence, qui est une condition nécessaire et non suffisante, plutôt que celle de succession pour modéliser l'ordonnancement des activités. Ainsi, si l'activité de décision est déclenchée, cela suppose qu'un événement a été détecté, que des simulations ont été réalisées sur des données préalablement collectées et que l'interprétation des résultats a révélé un risque qui a occasionné le déclenchement d'une alerte. L'utilisation de la relation de succession entre les activités est plus complexe car la succession n'est pas systématique. En effet, il faudrait ajouter d'autres conditions aux conditions d'occurrence d'activité précédentes (par exemple, une condition temporelle, liée à l'état de certaines entités ou encore liée à la disponibilité de ressources comme des données complémentaires).

\begin{tabular}{|c|c|c|}
\hline Activités & Précédent & Partie-de \\
\hline Veille & - & - \\
\hline Surveillance & - & Veille \\
\hline Analyse & Surveillance & Veille \\
\hline Décision & Analyse & Veille \\
\hline Evaluation & Décision & Veille \\
\hline Détection & - & Surveillance \\
\hline Collecte & Détection & Surveillance \\
\hline Simulation & Collecte & Analyse \\
\hline Interprétation & Simulation & Analyse \\
\hline Alerte & Interprétation & Analyse \\
\hline $\begin{array}{l}\text { Définition des plans } \\
\text { d'action }\end{array}$ & Alerte & Décision \\
\hline $\begin{array}{l}\text { Evaluation des plans } \\
\text { d'action }\end{array}$ & $\begin{array}{l}\text { Définition des plans } \\
\text { d'action }\end{array}$ & Décision \\
\hline $\begin{array}{l}\text { Sélection de plans } \\
\text { d'action }\end{array}$ & $\begin{array}{l}\text { Evaluation des plans } \\
\text { d'action }\end{array}$ & Décision \\
\hline $\begin{array}{l}\text { Mise en œuvre de plan } \\
\text { d'action }\end{array}$ & $\begin{array}{l}\text { Sélection de plans } \\
\text { d'action }\end{array}$ & Décision \\
\hline $\begin{array}{l}\text { Définition des } \\
\text { stratégies d'évaluation }\end{array}$ & $\begin{array}{l}\text { Mise en œuvre de plan } \\
\text { d'action }\end{array}$ & Evaluation \\
\hline $\begin{array}{l}\text { Mise en œuvre de } \\
\text { l'évaluation }\end{array}$ & $\begin{array}{l}\text { Définition des } \\
\text { stratégies d'évaluation }\end{array}$ & Evaluation \\
\hline
\end{tabular}

Tableau 1 : Relations de précédence et de composition des activités de la veille épidémiologique

\section{Formalisation et opérationnalisation des processus de la veille épidémiologique}

Le niveau logique requis pour faire des raisonnements sur l'ontologie des processus est la logique du premier ordre. L'expression de la sémantique des processus en logique permet d'automatiser certaines formes de raisonnement sur ce modèle, comme la classification automatique et les inférences.

Les langages existants ${ }^{1}$ de formalisation des processus sont essentiellement conçus pour la spécification des processus de manufacture et d'entreprise. Pour rendre opérationnels les modèles des processus de la veille [1] et d'automatiser le raisonnement sur ces modèles, nous avons choisi une formalisation utilisant le langage de spécification de processus $\mathrm{PSL}^{2}$ [12] qui est fondée sur la logique du premier ordre. Ce langage fournit un ensemble de primitives logiques permettant de représenter les

${ }^{1}$ Cyc, DDPO, oXPDL, m3po et m3pl, PSL, etc.

${ }^{2}$ http://www.mel.nist.gov/psl/ - The PSL was developed at the National Institute of Standards and Technology (NIST), and is approved as an international standard in the document ISO 18629. 
séquences possibles d'un ensemble d'activités, leurs conditions de transition, leurs conditions d'occurrence, les effets de leurs occurrences et les entités participant dans leurs occurrences. Ces primitives logiques sont spécifiées dans une ontologie décrivant formellement les composants et leurs relations qui entrent dans la description d'un processus. L'expression de la sémantique du langage en logique du premier ordre permet ainsi d'automatiser le raisonnement sur la description des processus. La définition formelle de la sémantique des primitives de PSL permet de contraindre leur interprétation. Par conséquent, l'usage du langage PSL pour la spécification d'un processus permet d'avoir un formalisme (une interprétation) unique (non ambigüe) des modèles de processus, facilitant ainsi leur échange entre les applications.

Parmi les primitives de base de PSL figurent les activités, les occurrences des activités, les objets et les intervalles de temps. Pour la formalisation des processus, nous utilisons les notions d'activité et d'occurrence d'une activité de PSL pour modéliser respectivement les notions de processus et d'événement présentées. Rappelons que les événements sont modélisés comme des occurrences des processus, une occurrence pouvant être comprise ici comme une instanciation. PSL est structuré en deux catégories: (i) "Core Theories" décrivant un ensemble primitif de concepts (object, activity, activityoccurrence, timepoint), relations (occurrence_of, participates_in, between, before, exists_at, is_occurring_at), constantes (inf + , inf-) et fonctions (begin of, end of), et (ii) "Definitional Extensions" étendant les éléments primitifs du langage pour définir des concepts et des relations plus complexes. Les primitives de PSL utilisées dans nos formalisations sont détaillées dans http://www.mel.nist.gov/ps1/.

\section{Patron de l'ontologie IDMPO-Core}

Le patron de l'ontologie noyau de ce processus générique de la veille épidémiologique des maladies infectieuses est un patron de structuration fournissant une organisation des processus de base de la veille épidémiologique généralisable à une application de veille d'une maladie infectieuse spécifique. Les détails de la modélisation du patron sont présentés dans [1]. Bien que la modélisation des processus soit caractérisée par la prise en compte de la dimension temporelle nous ne la traitons dans ce travail. La généralisation de ce patron permettrait de concevoir une ontologie prenant en compte cette dimension.

La primitive SUBACTIVITY-PRECEDES est utilisée pour l'ordonnancement des sous-activités d'une activité complexe. Contrairement à NEXT-ACTIVITY, il autorise l'occurrence d'une autre activité entre deux activités qu'elle relie dans une activité complexe. SUBACTIVITYPRECEDES laisse plus de possibilités à une redéfinition des activités dans le cadre d'une spécialisation ou d'une réutilisation du patron de l'ontologie IDMPO-Core ${ }^{3}$. La relation de composition entre les activités est modélisée avec la primitive SUBACTIVITY. L'Expression 1 donne un extrait de l'ontologie IDMPO-Core qui montre la définition de la relation de précédence entre quelques activités de la veille.

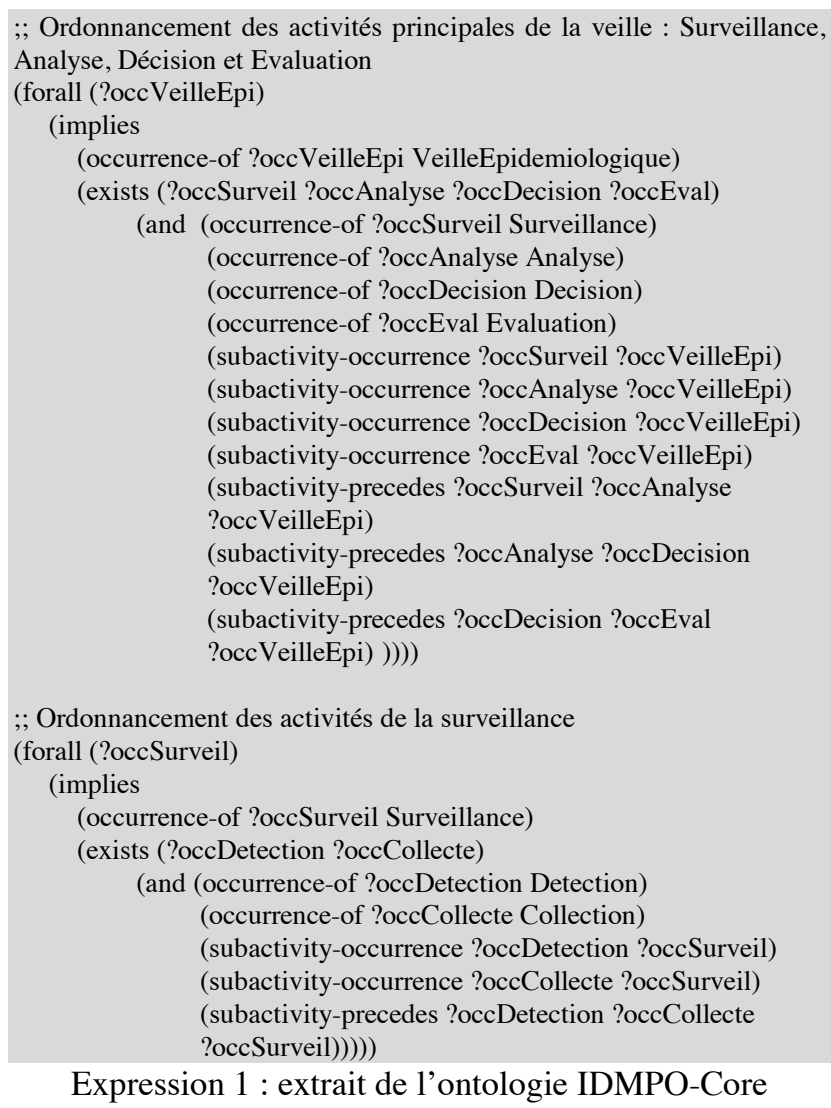

\section{Application du patron au processus de la veille de la schistosomiase au Sénégal}

Les éléments (processus, états, objets et axiomes) spécifiques à la veille de la schistosomiase ont été modélisés dans l'ontologie des processus de la veille de la schistosomiase $\left(\mathrm{SCHISTOMPO}^{4}\right)$. Les éléments généraux sont importés depuis l'ontologie noyau des processus de la veille épidémiologique (IDMPO). L'Expression 2 donne un extrait de la formalisation du processus de veille de la schistosomiase. Par exemple, le système de veille doit systématiquement détecter toutes les occurrences de

\footnotetext{
${ }^{3}$ IDMPO-Core : https://github.com/gaoussoucamara/idoschisto/blob/master /IDMPO-Core.kif

${ }^{4}$ SCHISTOMPO :

https://github.com/gaoussoucamara/idoschisto/blob/master /SCHISTOMPO.kif
} 
pluie car elles constituent des facteurs de risque de présence ou de variation de la densité des points d'eau, donc facteur de risque de propagation de la schistosomiase.

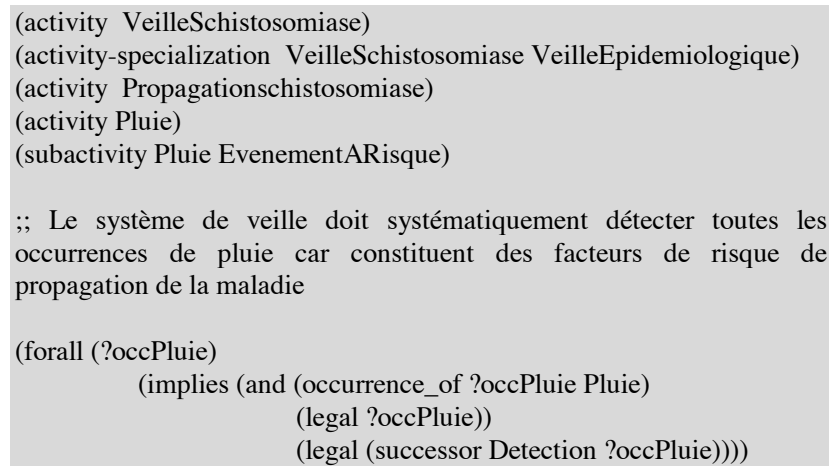

Expression 2 : extrait de l'ontologie SCHISTOMPO

\section{Conclusion et discussion}

Nous avons décrit dans cet article la notion de processus de veille de façon générale et sa spécialisation au processus de la veille épidémiologique des maladies infectieuses. En complément au travail de modélisation déjà entamé dans [1], ce papier vient enrichir cette modélisation et proposer sa formalisation et son opérationnalisation. Le choix de la logique requise et du langage d'opérationnalisation ont été justifié par le besoin d'automatisation du processus de la veille et de partage des modèles de processus obtenus.

L'ontologie des processus de veille épidémiologique est conçue sous forme de patron de structuration. Elle fournit les éléments de base (entités et axiomes) pour guider la construction d'une ontologie des processus de veille épidémiologique d'une maladie infectieuse spécifique. Nous avons testé ce patron en l'étendant pour la construction de l'ontologie de la veille de la schistosomiase au Sénégal.

Le choix de PSL est surtout guidé par notre objectif de construire une ontologie des processus. Cette ontologie nécessite une représentation formelle, fondée donc sur un formalisme logique. PSL est conçue pour la formalisation ontologique des processus et ses primitives sont décrites en logique du premier ordre pour favoriser l'automatisation des raisonnements sur les modèles obtenus. Néanmoins, il manque encore des outils pour la mise en œuvre du modèle obtenu. Aujourd'hui, la plupart des outils disponibles dans le domaine du web sémantique prennent en compte le formalisme de représentation OWL/XML alors que PSL est en CLIF. Pour pallier ces limites, nous proposons dans nos travaux futurs de fournir un mapping de PSL en OWL/XML pour garantir son opérationnalisation.

\section{Bibliographie}

[1] G. Camara, S. Despres, R. Djedidi, and M. Lo, "Modélisation ontologique de processus dans le domaine de la veille épidémiologique," in Actes de la conférence RFIA 2012, 2012.

[2] J. Christensen, "Epidemiological Concepts Regarding Disease Monitoring and Surveillance," Acta Vet. Scand., vol. 42, no. Suppl 1, p. S11, Mar. 2001.

[3] H. Inskip, V. Beral, P. Fraser, and J. Haskey, "Epidemiological monitoring: methods for analysing routinely-collected data," Sci. Total Environ., vol. 32, no. 3, pp. 219-232, Jan. 1984.

[4] D. Lewis, On the Plurality of Worlds. Oxford: Blackwell Publishers, 1986.

[5] P. Simons and J. Melia, "Continuants and Occurrents," Proc. Aristot. Soc. Suppl. Vol., vol. 74, pp. 59-75+77-92, 2000.

[6] D. Fensel, E. Motta, S. Decker, and Z. Zdrahal, "Using ontologies for defining tasks, problemsolving methods and their mappings," in Knowledge Acquisition, Modeling and Management, E. Plaza and R. Benjamins, Eds. Springer Berlin Heidelberg, 1997, pp. 113-128.

[7] R. Mizoguchi, J. Vanwelkenhuysen, and M. Ikeda, "Task ontology for reuse of problem solving knowledge," in Towards Very Large Knowledge Bases, N. J. I. Mars, Ed. IOS Press, 1995, pp. 4659.

[8] F. Buton, "De l'expertise scientifique à l'intelligence épidémiologique: l'activité de veille sanitaire," Genèses 20064, pp. p. 71-91, 2006.

[9] "Recommandations de deontologie et bonnes pratiques en epidemiologie," Rev. Epidémiologie Santé Publique, vol. 56, no. 3, Supplement, pp. S121-S148, juillet 2008.

[10] G. Camara, S. Després, R. Djedidi, and M. Lo, "Vers une ontologie des processus de propagation des maladies infectieuses," in Actes des 23èmes journées francophones d'ingénierie des connaissances, Paris, France, 2012, pp. 99-114.

[11] J.-L. Le Moigne, La modélisation des systèmes complexes. Paris: Dunod, 1990.

[12] M. Gruninger, "Using the PSL Ontology," in Handbook of Ontologies, S. Staab, Ed. Berlin: Springer-Verlag, 2009, pp. 419-431. 